\title{
Alternative myosin hinge regions are utilized in a tissue-specific fashion that correlates with muscle contraction speed
}

\author{
Venetia L. Collier, William A. Kronert, Patrick T. O'Donnell, Kevin A. Edwards, \\ and Sanford I. Bernstein ${ }^{1}$ \\ Biology Department and Molecular Biology Institute, San Diego State University, San Diego, California 92182 USA
}

By comparing the structure of wild-type and mutant muscle myosin heavy chain (MHC) genes of Drosophila melanogaster, we have identified the defect in the homozygous-viable, flightless mutant $M$ Mh ${ }^{10}$. The mutation is within the $3^{\prime}$ splice acceptor of an alternative exon (exon 15a) that encodes the central region of the MHC hinge. The splice acceptor defect prevents the accumulation of mRNAs containing exon 15a, whereas transcripts with a divergent copy of this exon (exon 15b) are unaffected by the mutation. In situ hybridization and Northern blot analysis of wild-type organisms reveals that exon 15b is used in larval MHCs, whereas exons $15 \mathrm{a}$ and/or 15b are used in adult tissues. Because $M \mathbf{M c}^{10}$ mutants fail to accumulate transcripts encoding MHC protein with hinge region a, analysis of their muscle-specific reduction in thick filament number serves as a sensitive assay system for determining the pattern of accumulation of MHCs with alternative hinge regions. Electron microscopic comparisons of various muscles from wild-type and $M \mathrm{Mc}^{10}$ adults reveals that those that contract rapidly or develop high levels of tension utilize only hinge region a, those that contract at moderate rates accumulate MHCs of both types, and those that are slowly contracting have MHCs with hinge region $b$. The presence of alternative hinge-coding exons and their highly tissue-specific usage suggests that this portion of the MHC molecule is important to the isoform-specific properties of MHC that lead to the different physiological and ultrastructural characteristics of various Drosophila muscle types. The absence of other alternative exons in the rod-coding region, aside from those shown previously to encode alternative carboxyl termini, demonstrates that the bulk of the myosin rod is not involved in the generation of isoform-specific properties of the MHC molecule.

[Key Words: Myosin heavy chain; Drosophila; muscle mutant; alternative RNA splicing]

Received January 12, 1990; revised version accepted March 6, 1990.

Muscle myosin heavy chain $(\mathrm{MHC})$ is a contractile protein whose rod-like domain forms the backbone of thick filaments and whose globular head domain acts as a bridge to actin-containing thin filaments (for review, see Harrington and Rodgers 1984). ATP-dependent deformation of the myosin cross-bridge results in muscle contraction. Functionally different muscles usually accumulate different isoforms of MHC (for review, see Emerson and Bernstein 1987). The amino acid variations among MHC isoforms may impart alternative myosin ATPase activities, actin or myosin light-chain-binding affinities, or thick filament assembly properties. Drosophila melanogaster is unusual in that a single muscle MHC gene, rather than a multigene family, encodes all forms of muscle MHC via alternative RNA splicing (Bernstein et al. 1986; Rozek and Davidson 1986; Was-

${ }^{1}$ Corresponding author. senberg et al. 1987; George et al. 1989; Hess et al. 1989). Analysis of the location of these alternative exons and their tissue-specific pattern of expression should help to elucidate the regions of the protein that impart isoformspecific properties to the MHC molecule. Alternative splicing at the $3^{\prime}$ end of the MHC transcript has been well documented, and it results in the production of proteins with alternative carboxyl termini (Bernstein et al. 1986; Rozek and Davidson 1986). MHC proteins containing one carboxyl terminus accumulate in larval muscles and in some adult muscles, whereas those containing the other carboxyl terminus are mostly found in adult thoracic musculature, as well as in some muscles of the adult head (Bernstein et al. 1986; Kazzaz and Rozek 1989).

The Drosophila MHC locus is haploinsufficient for flight muscle function (Bernstein et al. 1983), and mutations in the MHC gene that induce a flightless pheno- 
type have been isolated (Mogami and Hotta 1981; Mogami et al. 1986). A large proportion of these mutations are dominant flightless and homozygous lethal; the recessive lethality of these mutations likely arises as a result of disrupting MHC synthesis in all muscle types (Mogami et al. 1986; O'Donnell and Bernstein 1988). In contrast to these recessive-lethal alleles, several homozygous-viable MHC mutations cause muscle-specific reductions in MHC accumulation (Chun and Falkenthal 1988; O'Donnell et al. 1989). These mutations likely affect alternative exons that are used in muscles whose functions are not essential to viability.

Here, we report how the analysis of one of these mutants, $M h c^{10}$, led us to examine alternative splicing in the rod-coding region of the Drosophila MHC gene. MHC gene and cDNA sequence analysis indicates that the hinge domain of the rod is encoded by two mutually exclusive alternative exons. We found that the homozygous-viable $M \mathrm{Mc}^{10}$ mutation is a defect in the splice acceptor of one of these exons. Ultrastructural analyses of mutant and wild-type organisms, along with in situ hybridization studies, indicate that some muscles contain MHCs with one hinge region, whereas other muscles have MHCs of both types. These observations suggest the MHC hinge plays a key role in the musclespecific function of the myosin molecule.

\section{Results \\ Analysis of the molecular defect in the $\mathrm{Mhc}^{10}$ allele}

The homozygous-viable, dominant flightless mutant $M h c^{10}$ fails to accumulate MHC RNA, MHC protein, or thick filaments in the indirect flight muscles (IFM) and jump muscles of the adult and has reduced levels of MHC in leg muscles (O'Donnell et al. 1989). We were interested in determining the molecular lesion responsible for these defects and reasoned that it was probably associated with an alternative exon that was specifically included in transcripts of the affected muscles. We molecularly cloned the mutant MHC gene and sequenced the region containing the alternatively spliced 3 ' penultimate exon, which had been shown previously to be utilized in the affected muscle tissues (Bernstein et al. 1986; Kazzaz and Rozek 1989). However, no defects in the coding region or splice junctions of this or its flanking exons were found.

Having failed to detect a mutation in the penultimate exon of the $M h c^{10}$ allele, we examined upstream regions of the MHC gene to search for additional sites of alternative splicing. By comparison of the deduced amino acid sequence to previously sequenced MHCs, we were able to derive the exon/intron structure of the Drosophila MHC rod-coding region (Fig. 1A). The vast majority of the rod is encoded by constitutive exons, and the rod displays the expected 28 -amino-acid residue repeat that is important to the assembly properties of the myosin molecule (McLachlan and Karn 1982). Examination of the gene sequence, along with restriction map- ping and sequencing of several cDNA clones, revealed only one additional site of alternative splicing, which is within the hinge-coding region. Two 79-nucleotide alternative exons (exons 15a and 15b, according to the notation of George et al. 1989) are used in a mutually exclusive manner and encode amino acid sequences that differ by $72 \%$ (Fig. 1B). Sequence analysis of these exons in $M h c^{10}$ DNA revealed a single-base-pair change that mutates the consensus $3^{\prime}$ splice site of exon 15a from CAG to CAA (Fig. 1C).

Stage- and tissue-specific use of the alternative hingecoding regions

We examined the stage-specific usage of the alternative hinge-coding exons by preparing antisense RNA probes and hybridizing them to electrophoretic gel blots of RNA isolated from larvae and pupae (Fig. 2). At the larval stage, two size classes of transcripts $\mid 6.1$ and 6.6 $\mathrm{kb}$ ) accumulate in the wild type; these arise from the use of alternative polyadenylation sites and exclusion of the $3^{\prime}$ penultimate exon (Bernstein et al. 1986; Rozek and Davidson 1986). Both larval transcript classes hybridize to exon $15 b$, but neither hybridizes to exon 15a. The pattern for $M h c^{10}$ larvae is identical to that of wild type. At the pupal stage, both larval size classes of mRNA accumulate in wild-type organisms, as do two additional transcripts $(6.6$ and $7.1 \mathrm{~kb})$ that result from inclusion of the 3 ' penultimate exon and polyadenylation at either of two sites (Bernstein et al. 1986; Rozek and Davidson 1986). Note that the smaller-sized pupal-specific mRNA containing the 3 ' penultimate exon comigrates with the larger sized mRNA lacking that exon. As in larvae, exon $15 \mathrm{~b}$ hybridizes to the 6.1- and 6.6-kb mRNAs in both the wild-type and mutant pupae. Exon 15a, however, which hybridizes to the 6.6- and 7.1-kb mRNAs in the wildtype, does not detectably hybridize to transcripts in $M h c^{10}$ pupae (Fig. 2C). Prolonged exposure of Northern blots probed with exon 15a (not shown) reveals that this exon is included in 6.6- and 7.1-kb mRNAs of $M \mathrm{Mc}^{10}$ at $\sim 5 \%$ the level of wild type.

The $95 \%$ reduction in exon $15 \mathrm{a}$-containing transcripts in the mutant likely arises because the $3^{\prime}$ splice junction of exon 15a has been mutated from CAG to CAA. Note, however, that the mutation results in the production of an AG dinucleotide (CAAG). This cryptic splice junction may be used with low efficiency as a result of the lack of a consensus pyrimidine before the AG junctional signal (unspliced transcripts would likely be degraded). It is also possible that the cryptic splice junction is used efficiently (Smith et al. 1989) but that the spliced transcripts are unstable due to the presence of a stop codon in exon 15a resulting from a translational frameshift /see Fig. 1C). Transcript instability caused by a truncated open reading frame has been demonstrated previously in other systems (Baserga and Benz 1988). Any truncated MHC proteins produced from the mutant mRNA would likely be degraded, as sarcomeric MHCs require their rod regions for stability (Dibb et al. 1985; O'Donnell and Bernstein 1988). 
GATGGAACCAACAGAAATCTAACCTATTTTCTTTCCCCCCACCCAATCCAAAATCTCAATACGAAAAAAAAACAdGTGTTCTTCCGCGCCGGTGTCCTGGGTCAGATGGAGGAGTTCCG GATGAGCGTCTGGGCAAGATCATGTCCTGGATGCAGGCATGGG

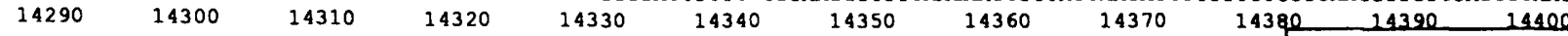

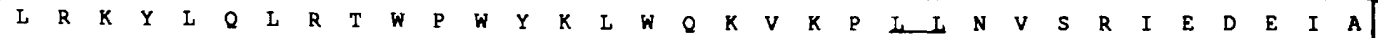
CTGCGCAAGTACCTGCAGCTCCGTACCTGGCCCTGGTACAAACTGTGGCAGAAGGTCAAGCCCCTCCTCAACGTCAGCCGCATCGAGGATGAGATTGCOGTGAGTATTCCCCAGAACGAA

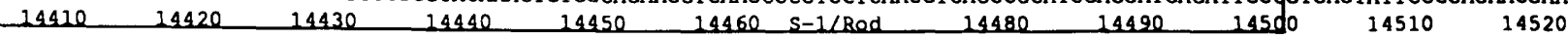
TGAGAACGAGACCAGCCTGGGTCTGGGGACTTAGTGGTCTTTGGTGATTCGATGCGATGAACTTGGACAGTACAGTTGCTGGTGGCAATCATTTGGTGACCGCAGCACGTTGATGTAGCC

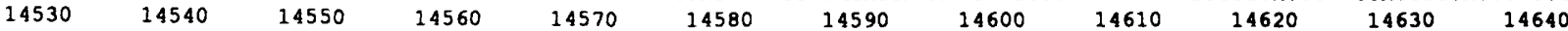

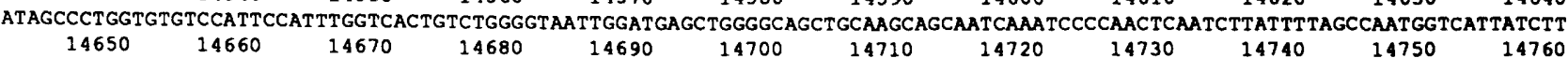
GTTTCT GATGCGAAATTCTTGCGCTTATTAACACACATCTTATACCTGCAGCAGGGGCCAATGAGAGGGAGAACCACCACCCACCCACTACCAGCTGCCCGAAACATATCGATACGATCT

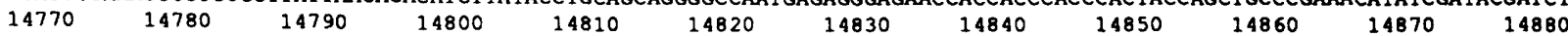
GATTCCAATCGCTGGCTAGCCAAAAATAGACGCTGTCATAATCTTTTCGCACT TTCGGGCCAAATGTGGCTCTATGTGTGTGCCCCAGGCGAATCATTTCGCTGGAATCCAACAACTCT
$14890 \quad 14900 \quad 14910$ TCACCTCTCACATTTTCATACACCCAAACCATCCCCCCATACGTACATTGTATGTATTTGGGTATGAATATTTTTCGAAAGTTATGACTTGTAGTTTGGGATCTATTCTTGGCCCTGCCA

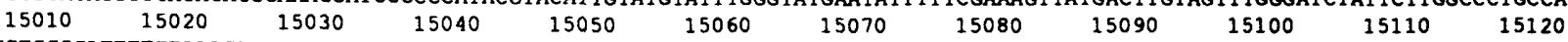
TATAGCTGGGGATTTTTTAAAGAGCCACAATTAGTTTGCCAATTAGCTTGCTAATTAAATAATAAAAAATGACCCGAGGATGCCCACGTATAGTTCCGATCCCCGATGAGTAATTTACCA

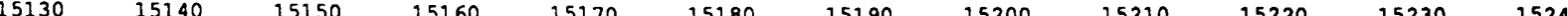
TGCATATGTACATCCATACGCACGATATTTCTAACCGATCTATATACCTAAGATGGTACACCTGCTGGTCCCGCCGCACAAGTGCACTTGATAGGCCCCATACCGACGCAATATAGCATT

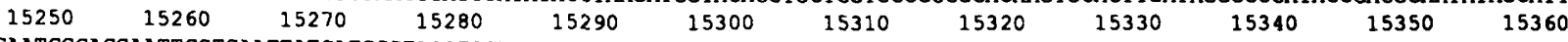
ATAGCAATGCCAGCAATTCGTGAATTATGATGGCTACCTCCTAACCAAGGCGATTACACACATGCCAAGTGGCAGCAGCTGCCATTTCGCTGCTCTGCTCTGCTCTGCTCTGGTGCCGTT

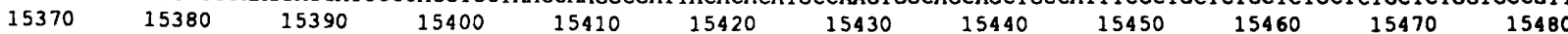
CTGCTCGCATCGGGGCGTTTTTGCTAATTTTATAGCCAAAGTGGCCCAGTGCATTTGCAGCAGCCATTCCAGTTGTATTTGACCAGTCGCCGTCGCGGTAACGCGTACCACAAAAAATCG

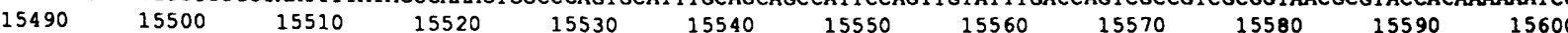

AACCCCTAACGAAGAACCAATCGATCTGAGCAAAAGAGCCACATTAGCCACAGAGCATCTTGACTGGCATCAAGATAACGAATTCAAATTGAAATTGTATTGTAGTTGACAATT GTGAAA

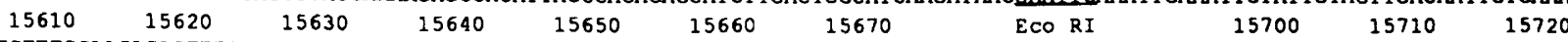

AGCCCCTTTCCAAGAGAGTT TCACATTTAATTAGGAGT TATTGAAAGTGCTTAATCATCAACATGGCCGATGAAAAGAAAGCCAAGAAAACGAAAAAGTCCACCGAATCGACCACACCCA

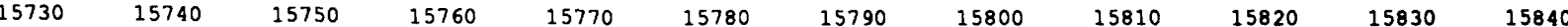
GTGCCACT GAGGAAGCAGCTCCAGCCGAAGCTGCTCCACCAGCAGAGACTGCAGAAGCTGCTCCTTCAGCCCGAATCGACCGCCGTCGAACCACCACAAAATCCCCAGCCAGCTGACGAG $\begin{array}{lllllllllllll}15850 & 15860 & 15870 & 15880 & 15890 & 15900 & 15910 & 15920 & 15930 & 15940 & 15950 & 15960\end{array}$

\section{exom 13}

CTCAGCTCCGGCAACAACCCTTCTAACCCATCAAATGCCTCTAATGACTTACAdCGTCTGGAGGAGAAGGCCAAGAAGGCTGAGGAACTGCATGCCGCTGAAGTGAAGGTGCGCAAGGAC

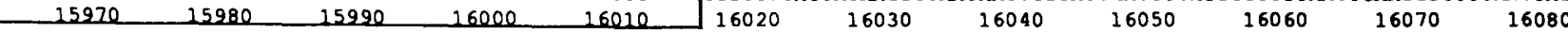
\begin{tabular}{lllllllllllllllllllllllllllllllllllllllll}
\hline L & E & A & L & N & A & K & L & L & A & E & K & T & A & L & L & D & S & L & S & G & E & K & $G$ & A & L & $Q$ & D & Y & $Q$ & E & $R$ & N & A & $K$ & L & T & A & $Q$ & K & $K$ \\
\hline
\end{tabular} CTCGAGGCCCTCAACGCCAAGCTTTTGGCTGAGAAGACCGCTCTGCTGGACTCCCTGTCCGGCGAGAAGGGTGCCCTGCAGGACTACCAGGAGCGCAACGCCAAGTTGACCGCCCAGAAC $\begin{array}{llllllllllll}16090 & 16100 & 16110 & 16120 & 16230 & 16140 & 16150 & 16160 & 16270 & 16180 & 16190 & 16200\end{array}$

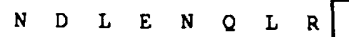

AACGACCTCGAGAACCAGCTGCGOGTAAGTATCCCATTAATCCCATACACTAAATCCCATTTATGCGATGGCATAAATCCAAGTGCCACGAAAGCGGAAAACGCGAAATTGATCAGAGGC

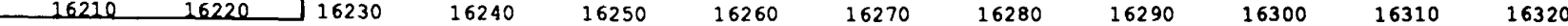
AATCGAGCATGAAAGTTGCTCGGCTATTTCTGGGCTCT GGCGCACTTGGCTAAATTTAGGAGTGACCCATCGCTAACT TCTCAGCCACTTAACACGCCCACACAATTTTGTTCAATTCCA

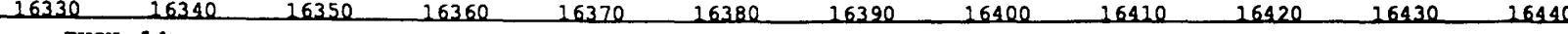
EXON 1

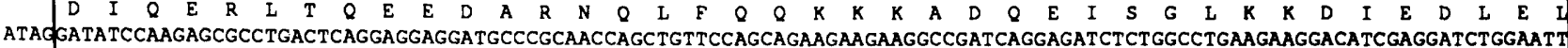
$\begin{array}{llllllllll}16450 & 16460 & 16470 & 16480 & 16490 & 16500 & 16510 & 16520 & 16530 & 16540\end{array}$

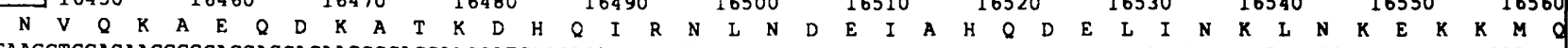
GAACGTCCAGAAGGCCGAGCAGGACAAGGCCACCAAGGATCACCAGATCCGCAACT TGAACGACGAGATCGCCCACCAGGATGAGCTCATCAACAAGTTGAACAAGGAGAAGAAGATGCA

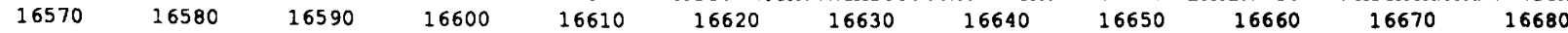

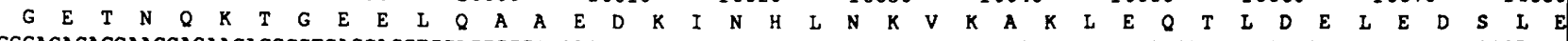
GGGAGAGACCAACCAGAAGACCGGTGAGGAGCTCCAGGCCGCCGAGGACAAGATCAACCACTTGAACAAGGTTAAGGCCAAGCTCGAGCAGACCCTCGATGAACTGGAGGATTCGCTGGA

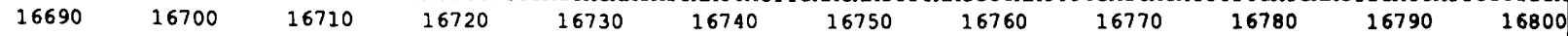

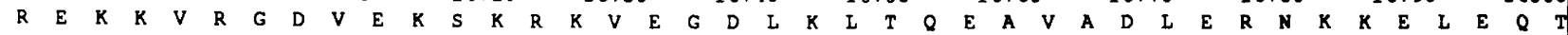
GCGCGAGAAGAAGGTGCGCGGCGATGTTGAGAAGTCCAAGCGCAAGGTTGAGGGCGACCTCAAGCTCACCCAGGAGGCTGTTGCCGATCTGGAGCGCAACAAGAAGGAGCTCGAGCAGAO

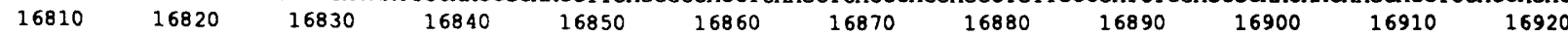

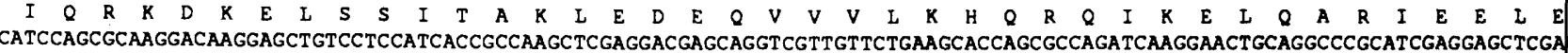

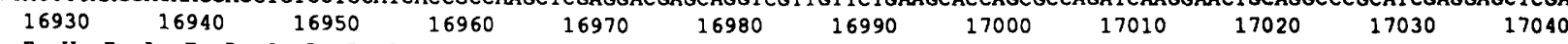

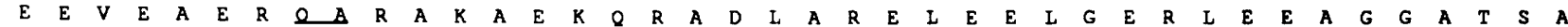
GGAAGAGGTCGAGGCTGAGCGCCAGGCCCGCGCCAAGGCTGAGAAGCAGCGCGCCGATCTGGCCCGCGAACTCGAGGAATTGGGCGAGCGTCTTGAGGAGGCTGGCGGTGCCACCTCTGC

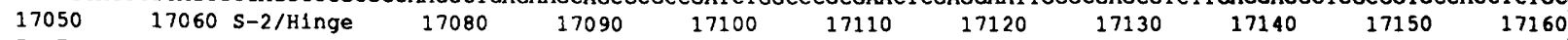

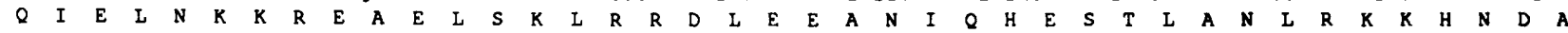
CCAGATTGAGCTCAACAAGAAGCGTGAGGCTGAGTTGAGCAAACTGCGTCGCGATCTTGAGGAGGCCAACATCCAGCACGAGTCCACCCTGGCTAACCTGCGCAAGAAGCACAACGATGC

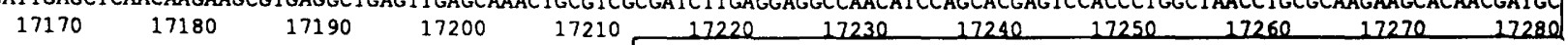

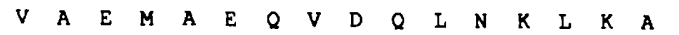

CGTCGCCGAGATGGCCGAGCAGGTTGATCAGCTCAACAAGCTGAAGGCTAATTAAGTATTGCGAATATTATTAGACTTCTGGCTAGCTTTTTCAGGTGCCAACGCTATCGAGATAGAGAG

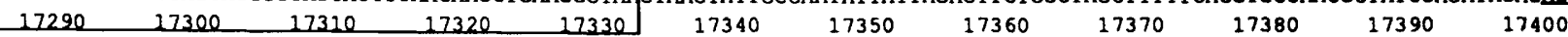

Figure 1. Part I (See p. 890 for legend.) 
ATCTTGAAGGATCTACAGTTTACAGTCTTCTTTCGAAGAGCTTTGGTTGCCGAACCCAACATAATAGATATTTTGCTCTTTTTTACCCATTTGCTAATCCAGGGCTGAACACGATCGCCA \begin{tabular}{|lllllllllllll} 
BglII & 17420 & 27430 & 17440 & 17450 & 17460 & 17470 & 17480 & 17490 & 17500 & 17510 & PVu I
\end{tabular}

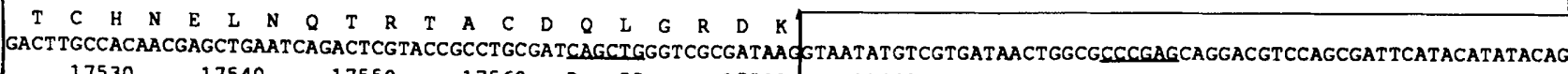

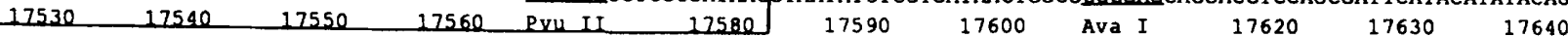

ATCCATACACTGAACACAGCATGTTCCAACCAACAAATAAAAAAAAAA TGCTACCACACAACTACGTACAAACGAACTACGTCTGTGTGTCCCTCTATCTCTCTCTCTCTCTGTCTAATG $\begin{array}{llllllllllll}17650 & 17660 & 17670 & 17680 & 17690 & 17700 & 17710 & 17720 & 17730 & 17740 & 17750 & 17760\end{array}$ AAATTGAAGGTTATTTGGAAAAAAGGTTCAACGCAAGGAAAGCAATTCTCTCGCTTCTCGCCTCTGTATTTGTCTCTGTCTCTGTCTTTATCCCCCACAAAACATGAACTAACCCGTGAA $\begin{array}{llllllllllll}17770 & 17780 & 17790 & 17800 & 17810 & 17820 & 17830 & 17840 & 17850 & 17860 & 17870 & 17880\end{array}$

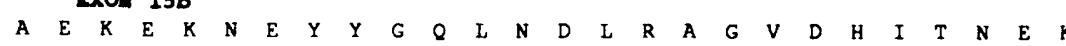

ATACGAACCTCTGTGTCTCTATCTATCTGTCTAAACCAdGGCTGAGAAGGAGAAGAACGAGTACTACGGCCAGTTGAACGATCTGCGCGCCGGTGTCGACCACATTACCAACGAGAAGGT $\begin{array}{llllllllllllll}17890 & 17900 & 17910 & 17920 & 17930 & 17940 & 17950 & 17960 & 17970 & \text { Sal I } & 17990 \quad 1800\end{array}$

ATTGAATTTGATCTCTACATATATTATTGCTCTCATCGCGATGCTGTAAATTTATATGCTGAAAGTTCTAGTTTAGCCACGTCTACTACATCTGTGTATAGTATCAACGTAGTTGCAACG

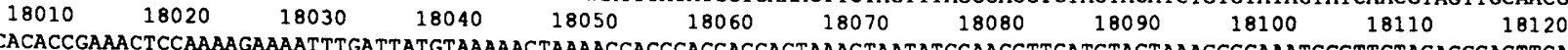
CAACCACACCGAAACTCCAAAAGAAAATTTGATTATGTAAAAACTAAAACGACCCACCACCACTAAAGTAATATCCAAGCTTGATCTACTAAACCCCAAATGCCTTGTACAGCCACTTGA $\begin{array}{llllllll}18130 & 18140 & 18150 & 18160 & 18170 & 18180 & 18190 & \text { HInd III } 18210\end{array}$ $18220 \quad 18230 \quad 18240$
Exco 16

CACGAAACCAAAATGCCACACT TACAATCAATTATGAATGTTCCCAATCCCCATCGTGAAAACTAATGAACATCCATTGCTTGAATCACTAATACGCTGCCCAGGAGAAGATCGCCAAGO

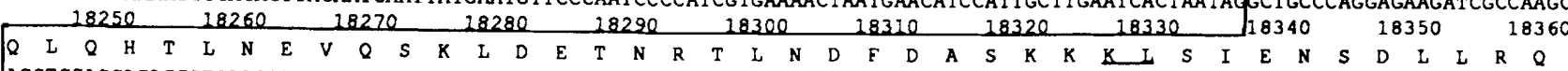
AGCTGCAGCACACCCTCAACGAGGTGCAGTCGAAACTGGATGAGACCAACAGGACTCTGAACGACTTCGATGCCAGCAAGAAGAAGCTGTCCATTGAGAACTCCGATCTGCTCCGCCAGC

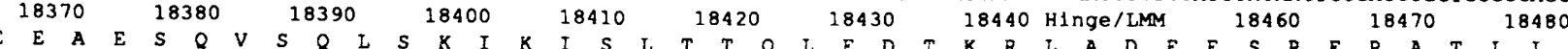
$\begin{array}{llllllllllllllllllllllllllllllllllllllllll}L & E & E & A & E & S & Q & V & S & Q & L & S & K & I & K & I & S & L & T & T & Q & L & E & D & T & K & R & L & A & D & E & E & S & R & E & R & A & T & L & L\end{array}$ TGGAGGAGGCCGAGTCCCAGGTGTCTCAGCTGTCCAAGATCAAGATCTCTCTGACCACCCAGTTGGAGGACACCAAGCGTCTGGCCGACGAGGAGTCGCGCGAGCGTGCCACCCTTTTGG

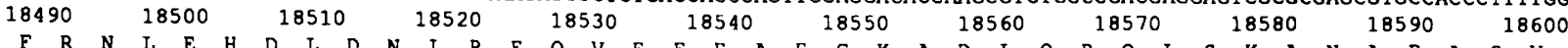

$\begin{array}{llllllllllllllllllllllllllllllllllllllllll}G & K & F & R & N & L & E & H & D & L & D & N & L & R & E & Q & V & E & E & E & A & E & G & K & A & D & L & Q & R & Q & I & S & K & A & N & A & E & A & Q & V\end{array}$ GCAAGTTCCGCAACTTGGAGCACGACCTGGACAATCTGCGCGAGCAGGTTGAGGAGGAGGCTGAGGGCAAGGCCGATCTGCAGCGCCAGCTGAGCAAGGCCAACGCTGAGGCCCAGGTGT

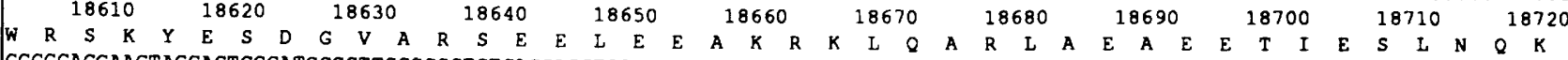
GGCGCAGCAAGTACGAGTCCGATGGCGTTGCCCGCTCTGAGGAGCTGGAGGAAGCCAAGAGGAAGCTGCAGGCCCGTTTGGCCGAGGCCGAGGACATCA

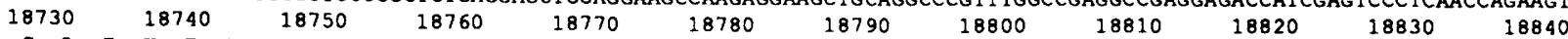

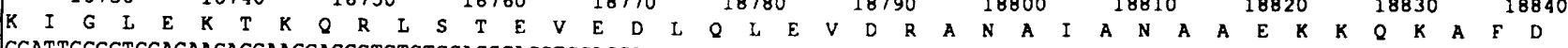
GCATTGGCCTGGAGAAGACCAAGCAGCGTCTGTCCACCGAGGTGGAGGATCTGCAGCTGGAGGTCGACCGTGCCAACGCCATTGCCAACGCTGCCGAGAAGAAGCAGAAGGCCTTCGACA

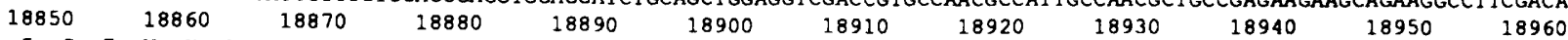

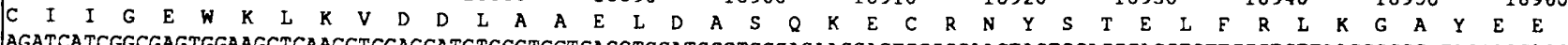
AGATCATCGGCGAGTGGAAGCTCAAGGTCGACGATCTGGCTGCTGAGCTGGATGCCTCCCAGAAGGAGTGCCGCAACTACTCCACCGAGCTGTTCCGTCTTAAGGGCGCCTACGAGGAGG

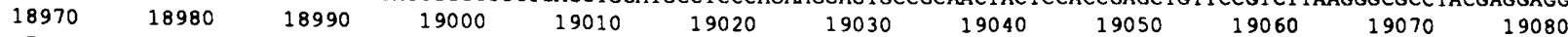

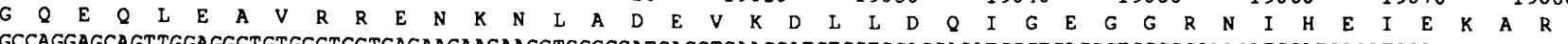
$\begin{array}{ccccccccccccccccccc}\text { GCCAGGAGCAGTTGGAGGCTGTGCGTCGTGAGAACAAGAACCTGGCCGATGAGGTCAAGGATCTGCTCGACCAGATCGGTGAGGGTGGCCGCAACATCCATGAGATCGAGAAGGCCCGCA } \\ 19090 & 19100 & 19110 & 19120 & 19130 & 19140 & 19150 & 19160 & 19170 & 19180 & 19190 & 19200\end{array}$

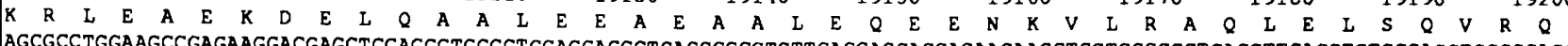
AGCGCCTGGAAGCCGAGAAGGACGAGCTCCAGGCTGCCCTCGAGGAGGCTGAGGCCGCTCTTGAGCAGGAGGAGAACAAGGTGCTCCGCGCTCAGCTTGAGCTGTCCCAGGTGCGCCAGG

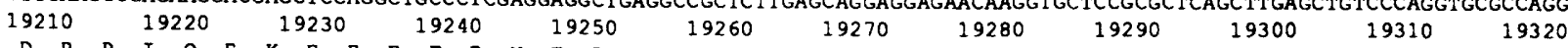

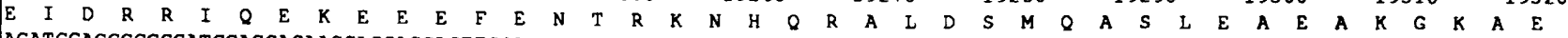

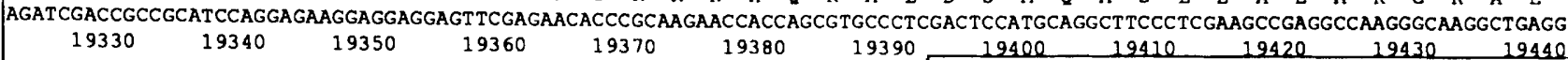
$\begin{array}{llllllllllllllllllllllllll}\text { A } & L & R & M & K & K & K & L & E & A & D & I & N & E & L & E & I & A & L & D & H & A & N & K\end{array}$ CCCTGCGCATGAAGAAGAAGCTGGAGGCTGACATCAACGAGCTTGAGATTGCTCTGGATCACGCCAACAAOGTAGGTTCAACCACTGATGCCTAGTCACACCGAGATGACTAACCTTAAT

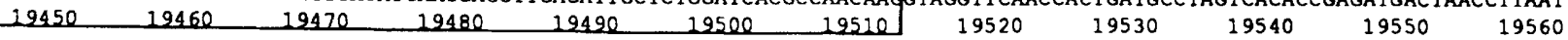

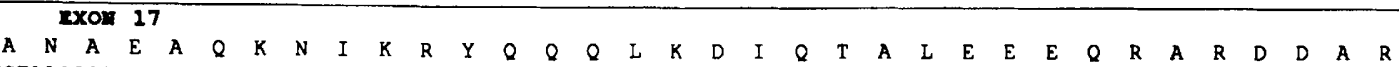
TCTTATCCTTTACTTTAgGCTAACGCCGAGGCCCAGAAGAACATCAAGCGTTACCAGCAGCAGCTGAAGGACATCCAGACTGCCCTCGAGGAGGAGCAGCGCGCCCGCGACGATGCCCGC

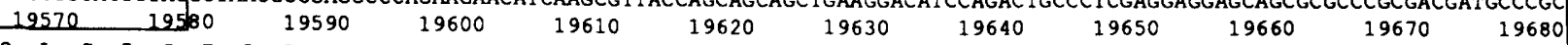

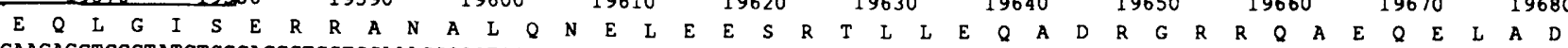
GAACAGCTGGGTATCTCCGAGCGTCGTGCAAACGCCCTCCAGAACGAACTGGAGGAGTCTCGCACTCTGCTGGAACAGGCCGACCGTGGCCGTCGCCAGGCCGAACAGGAGCTGGCCGAT

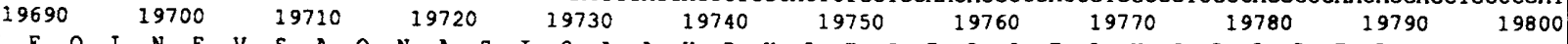

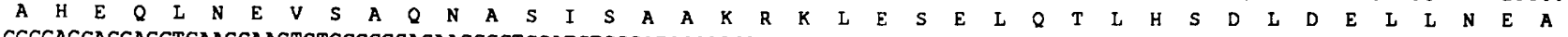
GCCCACGAGCAGCTGAACGAAGTGTCCGCCCAGAACGCCTCCATCTCCGCTGCCAAGAGGAAGCTGGAGTCCGAGCTGCAGACCCTGCACTCCGACCTGGACGAACTCCTGAACGAAGCC

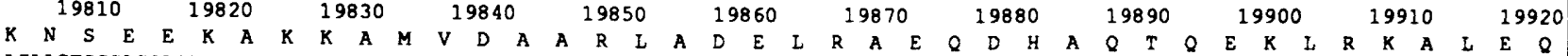
AAGAACTCCGAGGAGAAGGCCAAGAAGGCTATGGTCGATGCCGCCCGCCTGGCCGATGAGCTCCGCGCTGAGCAGGATCATGCCCAGACCCAGGAGAATTGAGGAAGGCCCTCGAGCAG

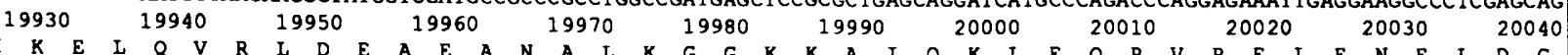

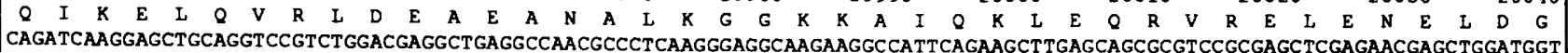

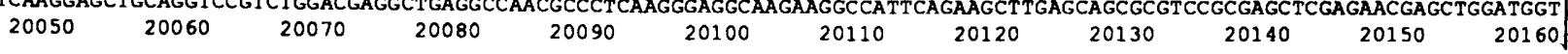

Figure 1. Part II (See p. 890 for legend.) 


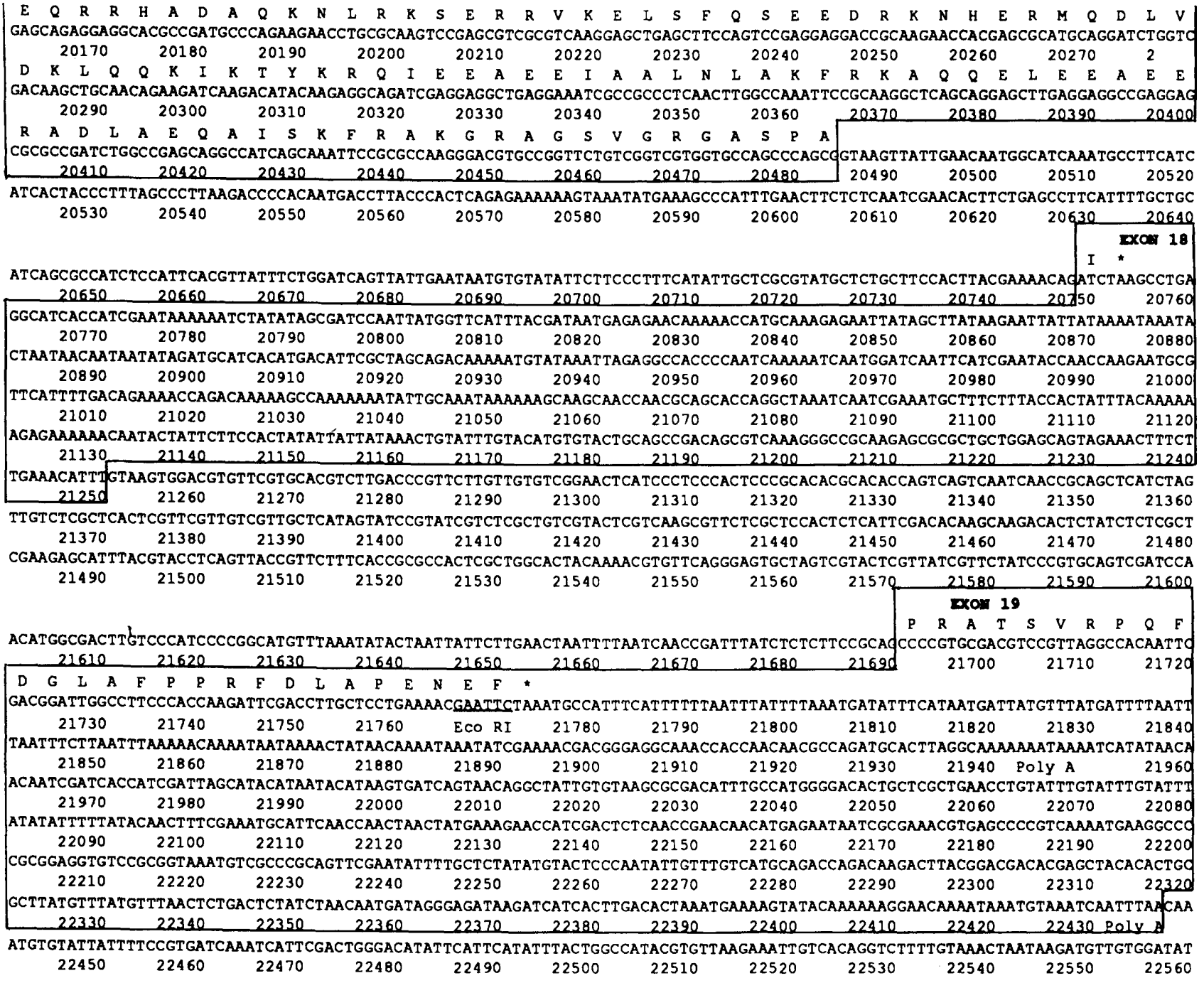

\section{$\boldsymbol{B}$}

Rat emb. MHC LKKKDFEYSOLQSKVEDEQTLSLQLQKKI KE LOAR IEELEEE IEAERATRAKTEKORSDYARELEELSERLEEAGGVTSTOIELNKKREAEF LKLRRDLEEATLOHE c. elegans LKKKESE LHSVSSRLEDEHALVSKLQRQIKDGQSRI SELEEELENERQSRSKADRAKSD LQRELEE LGEKLDEQGGATAAQVEVNKKREAE LAKLRRDLEEANMNHE Dros. MHC IQRKDKELSSITAKLEDEQVVVLKHQRQI KELQARIEELEEEVEAERQARAKAEKQRAD LARELEE LGERLEEAGGAT SAQI ELNKKREAELSKLRRDLEEANIQHE

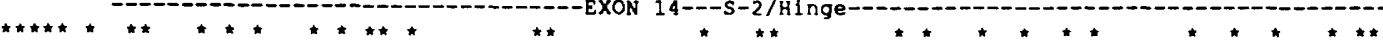
ATVATLRKKHADSAAE LAEQIDNLQRVKQKLEKEKSEFKLE IDDLSSSVESVSKSKANLEKICRTLEDQLSEARGKNEETQRSLSELTTQKSRLOTEAGE LSRQ NQLGGLRKKHTDAVAELTDQLDQLNKAKAKVE KDKAQAVRDAEDLAAQLDQET SGKLNNEKLAKQFE LQLTE LQSKADEQSRQLQDFT SLKGRLHSENGDLVRQ ST LANLRKKHNDAVAEMAEQVDQLNKLKAKAEHDRQI CHNE LNQTRTACDQLGRDKAAOEKIAKQLQHT LNEVQSKLDETNRT LNDFDASKKKCS I ENSDLLRQ

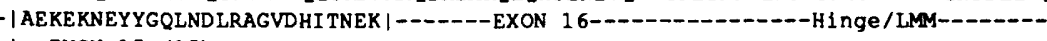
$\mid--$ EXON $15 a / 15 b----------1$

\section{C}

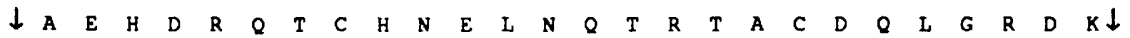
W1Id type TTTACCCATTTGCTAATCCAGGGCTGAACACGATCGCCAGACTTGCCACAACGAGCTGAATCAGACTCGTACCGCCTGCGATCAGCTGGGTCGCGATAAGGTAAT $\downarrow$ L

Mh $^{10}$ ITTACCCATTTGCTAATCCAAGGCTGAACACGATCGCCAGACTTGCCACAACGAGCTGAATCAGACTCGTACCGCCTGCGATCAGCTGGGTCGCGATAAGGTAAT

Figure 1. Part III (See following page for legend.) 
We used the antisense RNA probes for the hingecoding regions to determine the tissue specificity of alternative exon usage via in situ hybridization to wildtype organisms. As expected, exon 15b hybridized to transcripts in all larval muscles, whereas exon 15a failed to hybridize to larval muscle (not shown). In agreement with these observations, 23 independent cDNA clones isolated from a late embryo cDNA library (Brown and Kafatos 1988) all contained exon 15b. In adults, a more complex pattern emerges (Fig. 3). Exon 15a, but not exon $15 \mathrm{~b}$, is utilized in IFM and jump muscles; however, both exons are used in various head and leg muscles. The failure of $M h c^{10}$ mutants to accumulate mRNAs containing exon $15 \mathrm{a}$, along with the tissue-specific usage of this exon in wild-type flies, correlates well with our previous demonstration that $M h c^{10}$ pupae do not have detectable levels of MHC mRNA and protein in IFM and jump muscles and show reduced accumulation in leg muscles (O'Donnell et al. 1989).

\section{Ultrastructural analysis of the $\mathrm{Mhc}^{10}$ mutant is a highly sensitive assay for usage of alternative $M H C$ hinge regions}

From our in situ hybridization studies, it is clear that there is a tissue-specific pattern of accumulation of MHCs with alternative hinge regions. In situ hybridization is inadequate for determining the ratio in which alternative hinge-encoding $\mathrm{MHC}$ transcripts are coexpressed. However, the $M h c^{10}$ strain provides an opportunity to examine the ultrastructure of muscles lacking MHC with hinge region a, and one can thereby infer the relative proportions of MHCs with each of the alternative hinge regions. Only exon $15 \mathrm{a}$ is used in IFM and jump muscles (Fig. 3), and these muscles in $M h c^{10}$ flies do not accumulate MHC transcripts, MHC protein, or thick filaments (Fig. 4B; O'Donnell et al. 1989). In contrast, muscles of the upper leg (coxa) are only partially affected by the mutation. Intracoxal levator muscles (seen in cross section in Fig. $4 \mathrm{H}$ ) show an $\sim 50 \%$ reduction in thick/thin filament ratio compared to wild type (Fig. 4E). Intracoxal depressor muscles are more severely affected, as they lack rectangular myofibrils (Fig. 4F) and have only occasional thick filaments that are abnormal in shape (Fig. 4G). Finally, the dorsal body wall muscles of the abdomen appear completely normal in the mutant (Fig. 4K). On the basis of our in situ hybridization and electron microscopic evidence, we conclude that the severity of reduction in thick filament number in the mutant muscles correlates with the use of exon 15a in the wild-type muscle.

\section{Homozygous-viable MHC mutations that display different tissue-specific defects from $\mathrm{Mhc}^{10}$ are not within exon 15}

We characterized previously three other homozygous-viable $\mathrm{MHC}$ mutations $\left(M h c^{7}, M h c^{9}\right.$, and $\left.M h c^{11}\right)$ which, like $M h c^{10}$, prevent myosin accumulation in the IFM (O'Donnell et al. 1989; see also Chun and Falkenthal 1988|. Unlike $M h c^{10}$, the $M h c^{7}, M h c^{9}$, and $M h c^{11}$ mutations do not affect MHC accumulation in leg muscles and have less severe effects on thick filament accumulation in the jump muscle. The different tissue specificities of the $M h c^{7}, M h c^{9}$, and $M h c^{11}$ mutations suggested that they should not affect exon 15a. We cloned and sequenced exons $15 a$ and $15 b$ and their surrounding introns from these mutants and failed to find any differences from the wild-type sequence. These results indicate that the genetic lesions in the $M h c^{7}, M h c^{9}$, and $M h c^{11}$ alleles likely arise from mutations in alternative exons other than $15 \mathrm{a}$ and $15 \mathrm{~b}$.

\section{Discussion}

Our results indicate that there is remarkable complexity to the accumulation of transcripts encoding MHCs with alternative hinge regions in Drosophila. Embryonic and larval muscles as well as abdominal body wall muscles of the adult use only the hinge encoded by exon $15 \mathrm{~b}$. Adult leg muscles and the proboscis musculature apparently accumulate some MHCs containing each hinge

Figure 1. (A) DNA sequence analysis of the rod-coding region of the Drosophila muscle MHC gene. The nucleotide numbering system corresponds to that used previously (Wassenberg et al. 1987), and exon numbers are assigned on the basis of sequencing of the entire MHC gene (George et al. 1989; W. Kronert and S. Bernstein, unpubl.). Sequencing of the rod-coding region of wild-type genomic DNA and analysis of several cDNA clones revealed only a single set of alternative exons (15a and 15b). Exon/intron boundaries were determined by their homology to consensus splice sequences (Mount 1982), by comparison of the encoded amino acid sequence to MHCs of other organisms (Karn et al. 1983; Strehler et al. 1986), and by partial sequencing of a larval and a pupal cDNA clone. The borders of the hinge regions are denoted, and the amino acids that correspond to the proteolytic cleavage sites in vertebrate MHC protein (Strehler et al. 1986) are shown in boldface type. The penultimate exon of the gene (exon 18) is included in some adult transcripts, where it encodes a single carboxy-terminal amino acid. This exon is omitted from other MHC mRNAs, in which case exon 19 encodes the carboxyl terminus of the protein (see Fig. 2A; Bernstein et al. 1986; Rozek and Davidson 1986). (B) Comparison of the amino-acid-coding potential of Drosophila, rat embryonic skeletal muscle (Strehler et al. 1986), and C. elegans unc-54 MHC (Karn et al. 1983/ hinge regions. The region encoded by the alternatively spliced Drosophila exons is embedded in the central portion of the hinge. Asterisks (*) indicate when both the rat and nematode sequences contain the same amino acid as at least one of the Drosophila MHCs. $(C)$ Sequence comparison of exon $15 \mathrm{a}$ from wild type and the flightless mutant $M \mathrm{hc}^{10}$. The single-nucleotide change $(\mathrm{G} \rightarrow \mathrm{A})$ that eliminates the consensus $3^{\prime}$ splice junction (PyAG) is underlined. This also creates a nonconsensus $3^{\prime}$ splice junction (AAG) that may be used for splicing (see text). As shown, this would result in the production of a truncated protein due to shifting of the translational reading frame. Splice junctions are indicated by arrows. 
B

A

MHC TRANSCRIPTS:

COMMON FORMS

PUPALADULT-

SPECIFIC FORMS

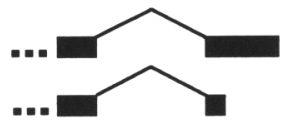

$6.6 \mathrm{~kb}$

$6.1 \mathrm{~kb}$
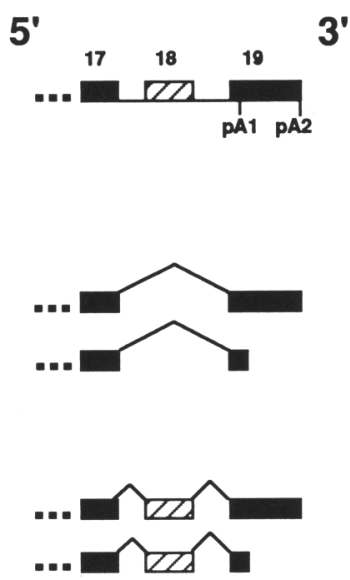

$7.1 \mathrm{~kb}$

$6.6 \mathrm{~kb}$

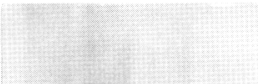

C

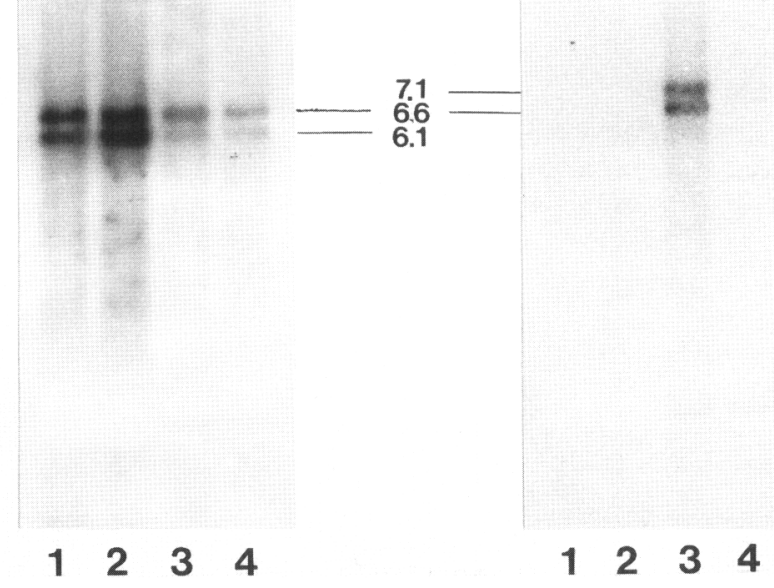

Figure 2. Hybridization of an RNA gel blot with probes specific to alternative exons 15a and 15b. $(A)$ Alternative splicing and polyadenylation patterns that give rise to the various size classes of MHC mRNA. Only the ${ }^{\prime}$ ' end of the gene is shown; alternative inclusion of the penultimate exon (exon 18) and polyadenylation at pA1 or pA2 result in four transcript classes. Alternative splicing of upstream exons does not affect the size of the mRNAs (Wassenberg et al. 1987; George et al. 1989; Hess et al. 1989). (B) Hybridization of the exon $15 \mathrm{~b}$ probe reveals transcripts of 6.1 and $6.6 \mathrm{~kb}$ in wild-type larval (lane 1) and pupal (lane 3 ) RNA, as well as in $M \mathrm{Mc}^{10}$ larval (lane 2) and pupal (lane 4) RNA. (C) Hybridization of the exon 15a probe to the same blot shown in $B$ (following removal of the exon $15 \mathrm{~b}$ probe) reveals MHC transcripts of 6.6 and $7.1 \mathrm{~kb}$ in the wild-type pupal RNA (lane 3) but no hybridization to larval RNA (lanes 1 and 2) or to RNA from $M h c^{10}$ pupae (lane 4). On longer exposures of this blot, a very minor amount of the 6.6- and 7.1-kb transcripts in the $M h c^{10}$ pupal lane could be observed (not shown).

type. Ultrastructural analysis of two different types of leg muscles in the $M \mathrm{Mc}^{10}$ mutant reveals that the ratio of the two MHCs can vary dramatically between such muscles. At the other end of the spectrum, the highly specialized IFM and jump muscles use solely exon 15a. In agreement with our results, George et al. (1989) showed by $\mathrm{S} 1$ nuclease mapping that only exon $15 \mathrm{a}$ is included in IFM transcripts, whereas exon $15 \mathrm{~b}$ is predominant in larvae and adult abdomen. Although it is possible that the tissue-specific inclusion of exon 15a or $15 \mathrm{~b}$ is serendipitous, we believe it is more likely that the alternative hinge regions encoded by these exons directly affect the functional properties of the MHC molecule.

The MHC rod has an $\alpha$-helical structure that permits the interaction of MHC molecules to form dimers and the interaction of MHC dimers to form thick filaments (McLachlan and Karn 1982). The two major helixbreaking domains of the rod are the hinge and the carboxy-terminal tailpiece. Interestingly, our results and those published recently by George et al. (1989) indicate that these are the only regions of the Drosophila MHC rod that are encoded by alternatively spliced exons. On the basis of our Northern blots and in situ hybridizations of both wild-type and $M h c^{10}$ organisms, as well as previous studies (Bernstein et al. 1986; George et al. 1989; Kazzaz and Rozek 1989|, it appears that the use of exon 15a generally correlates with the inclusion of the $3^{\prime}$ penultimate exon of the MHC gene /which encodes an alternative tailpiece). Thus, the hinge and tailpiece may act in concert with each other and, perhaps, with regions of the globular head encoded by alternative exons (Was- senberg et al. 1987; George et al. 1989; Hess et al. 1989|, to produce functional differences between MHC isoforms. Our results rule out the possibility that regions of the rod other than the hinge impart isoform-specific properties to the MHC molecule. This conclusion may apply to MHC isoforms encoded by multigene families as well.

Heterogeneity in the MHC rod or tailpiece might be expected to affect assembly properties of myosin molecules (Kuczmarski and Spudich 1980; McLachlan and Karn 1982; Kiehart et al. 1984), the morphology of thick filaments, and the number of thin filaments that assemble around each thick filament (Hayashi et al. 1983) and, perhaps, force generation (Ueno and Harrington 1986). Thin filament orbital number correlates roughly with use of exon 15b, it being lowest in IFM /which never utilizes exon 15b/ and greatest in larval and abdominal body wall muscles (which use only exon 15b). Hayashi et al. (1983) found that thick filaments assemble in vitro with specific numbers of thin filaments, depending on the type of myosin included in the thick filaments. Our results suggest that the MHC hinge and/ or carboxyl terminus may be implicated in this process. Another correlation we have observed is that muscles required to contract quickly (IFM) and/or generate high levels of tension (jump muscle) accumulate solely transcripts encoding hinge region a, whereas muscles that contract slowly (larval muscles and abdominal muscles of the adult) only accumulate hinge region b-coding transcripts. Muscles of an intermediate contraction speed (leg and proboscis muscles) contain some transcripts of each type. Alternative myosin hinges may 

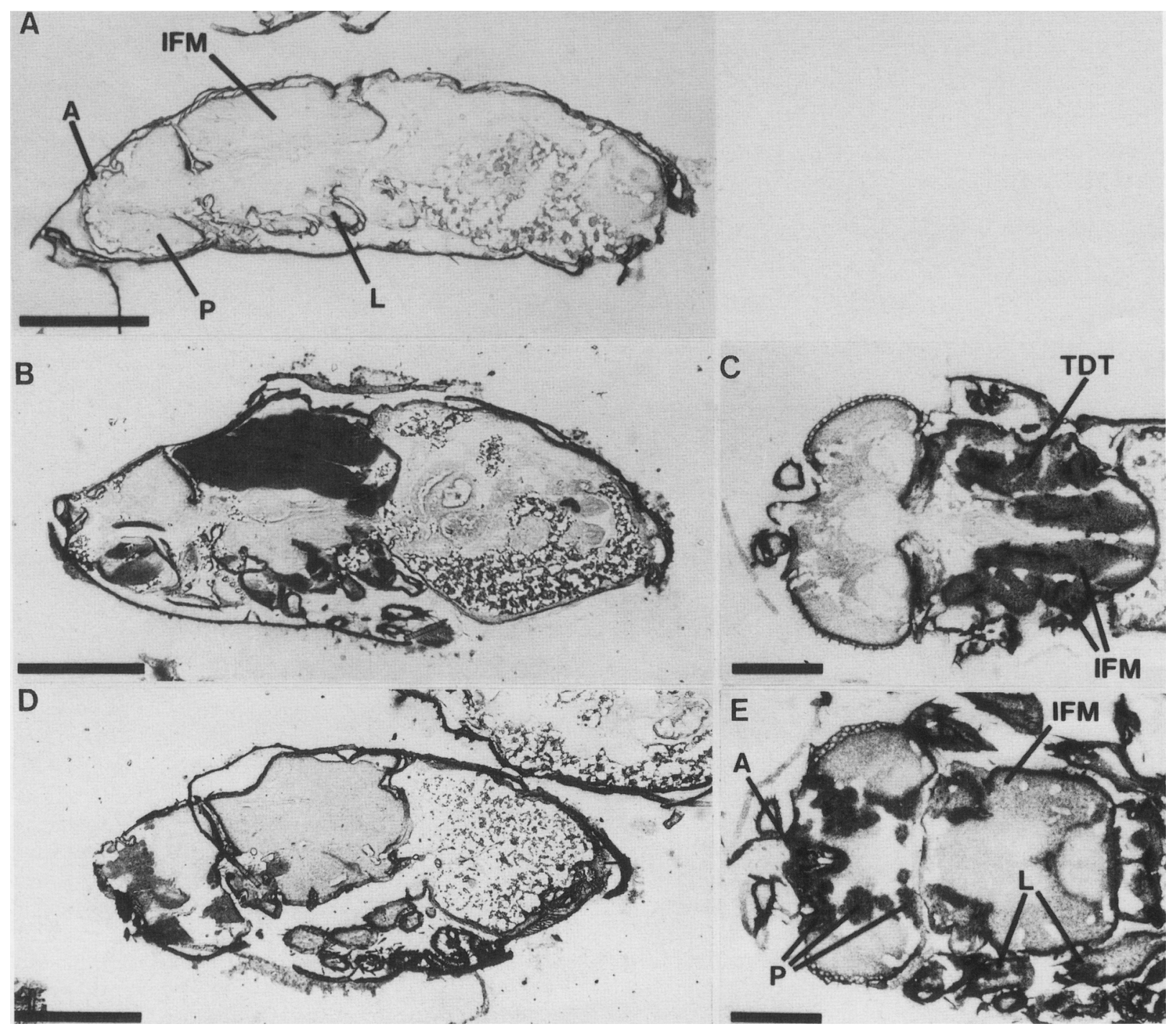

Figure 3. In situ hybridization of radiolabeled RNA probes to cryosections of wild-type Drosophila pupae. Anterior is to left in $A-E$. (A) A control (sense) probe transcribed from the MHC gene (exons 4-6; see O'Donnell et al. 1989) and hybridized to parasagittal section does not result in silver grain accumulation over muscle tissues. (IFM) Indirect flight muscle; $(\mathrm{P})$ proboscis muscles; (L) leg tissues; (A) antennal muscles. $(B)$ Exon 15a antisense probe hybridizes to IFM, some proboscis muscles, and leg muscles. $(C)$ Dorsal view of pupa hybridized with exon 15a antisense probe shows grains over IFM and jump muscle ([TDT) tergal depressor of the trochanter muscle]. $(D)$ Exon $15 \mathrm{~b}$ antisense probe fails to hybridize to IFM but clearly labels proboscis muscles. $(E)$ Exon $15 \mathrm{~b}$ probe hybridizes to proboscis (P), antennal (A), and leg muscles (L) but not to IFM. $(A, B$, and $D)$ Bar, $440 \mu ;(C$ and $E)$ bar, $250 \mu$.

therefore play a role in the generation of different levels of contractile force or speed. Indeed, Harrington and colleagues (Harrington and Rodgers 1984; Ueno and Harrington 1986) argue that force generation is a result of conformational changes within the hinge. However, Kishino and Yanagida (1988) and Spudich and colleagues (Hynes et al. 1987; Toyoshima et al. 1987) observe myosin force production or movement in vitro in the

Figure 4. Ultrastructures of muscles from wild-type and $M h c^{10}$ adults reveal the pattern of exon $15 \mathrm{a}$ usage. Reduced numbers of thick filaments result from failure to correctly splice exon $15 \mathrm{a}$ in the mutant. Wild-type IFM $(A)$ displays a highly ordered arrangement of six thin filaments surrounding each thick filament. In the mutant $|B|$, no normal thick filaments are observed. In $C$, the two muscle groups of the coxal segment of the leg, the intracoxal levators (IL), and the intracoxal depressors (ID) are indicated (Crossley 1978). The wild-type intracoxal depressor is shown in oblique section $(D)$ and has a regular myofilament lattice. This muscle in $M h c^{10}$ legs $(F)$ contains extremely disorganized myofibers whose myofilaments are in complete disarray. At higher magnification, thick filaments are seen only occasionally $(G)$, and these are abnormal in shape. $Z$ disc material $(Z)$ is poorly organized. The mutant intracoxal levator muscle $(H)$ shows an $\sim 50 \%$ decrease in the thick/thin filament ratio, with some disruption of the thick filament linear array seen in wild type $(E)$. In $I$, the dorsal body wall muscles $(D M)$ of the adult abdomen are shown. At higher magnification, wild-type $(J)$ and $M h c^{10}(K)$ dorsal body wall muscles have identical myofilament arrays. Glycogen granules (G), mitochondria (M), and sarcoplasmic reticulum $(S)$ are indicated. Bars for electron micrographs, $0.2 \mu$; bars for light micrographs $(C$ and $I), 16 \mu$. 
absence of the hinge-containing rod region. These experiments, although clearly demonstrating that the Sl globular head of $\mathrm{MHC}$ is capable of generating movement and force, do not rule out the possibility that the hinge modulates these properties directly or by changing the structure of the thick filament and/or the geometry of the interaction between thick and thin filaments. The presence of alternative hinge regions in Drosophila

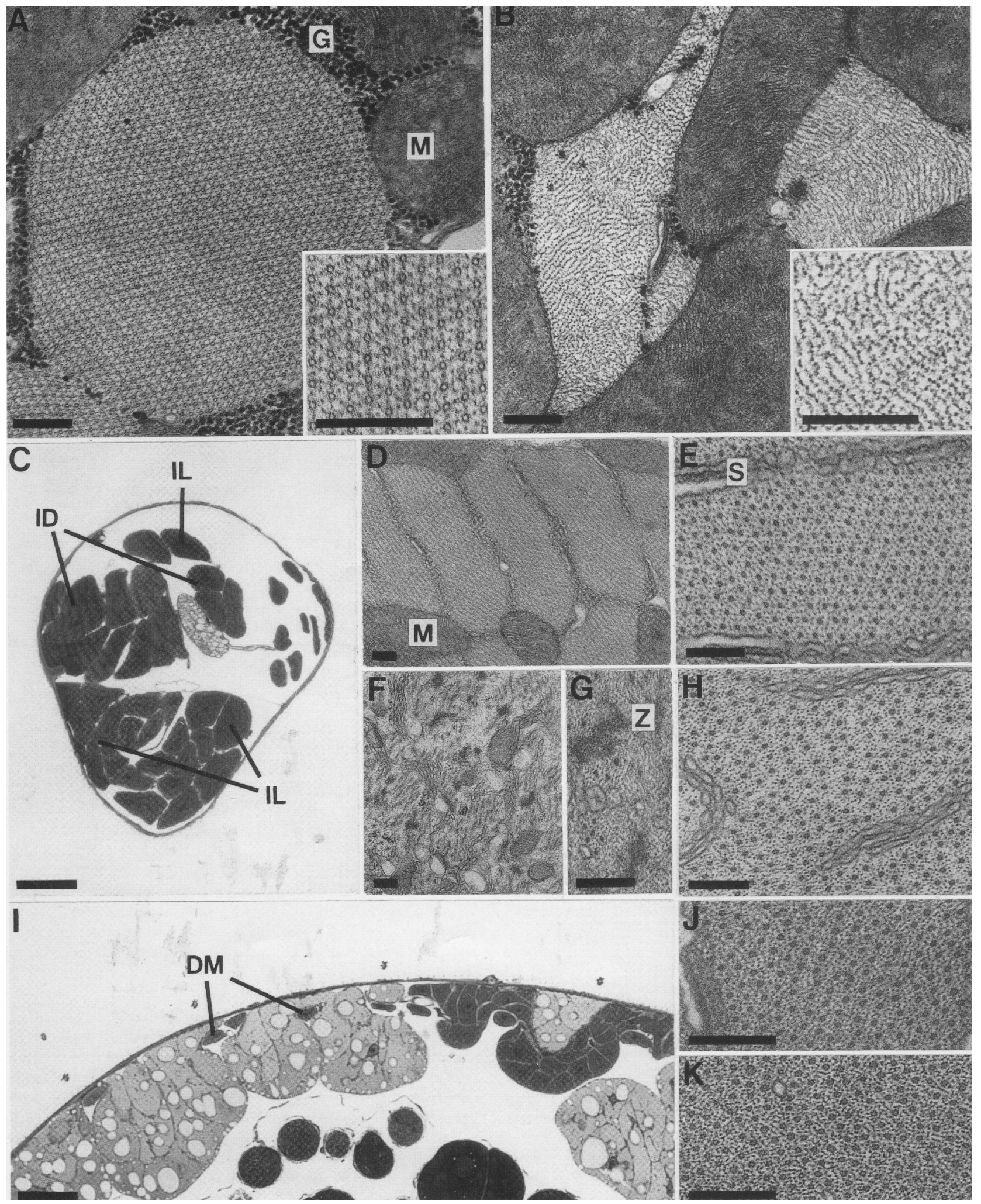

Figure 4. (See facing page for legend.) 
MHC and the general correlation with contraction speed and/or tension development suggest that the hinge may indeed have an effect on muscle contraction.

Use of the $M \mathrm{MC}^{10}$ null mutant in conjunction with MHC gene/cDNA fusions introduced by P-element-mediated germ line transformation should permit the analysis of IFM and jump muscles forced to express hinge $b$. Subsequent mechanical measurements of contraction speed and force would indicate the effect of the alternative hinges on these parameters. Likewise, ultrastructural examination would show whether the morphology of the thick filament or the thin filament orbital number is affected by the hinge region of the MHC molecule. This in vitro mutagenesis/gene transformation approach should therefore be a useful and unique way of analyzing MHC domain function in vivo.

\section{Materials and methods \\ Isolation and sequencing of genomic and cDNA clones}

Wild-type genomic MHC DNA (Bernstein et al. 1983) was subcloned into plasmid vectors prior to sequence determination. BamHI-digested DNA from the homozygous $M h c^{10}$ strain was cloned into $\lambda$ vector EMBL3 (Stratagene) and packaged into phage heads by use of the Gigapack extract (Stratagene). The recombinant library was screened by use of a nick-translated probe containing the $3^{\prime}$ end of the MHC gene. A positive phage containing a 16-kb BamHI fragment that encompasses the $3^{\prime}$ half of the MHC gene was isolated. Sequences were determined on plasmid subclones of genomic MHC DNA by the dideoxynucleotide chain-termination method (Hattori and Sakaki 1986) with synthetic oligonucleotide primers. Late embryonic cDNA clones were obtained by screening a cDNA plasmid library (Brown and Kafatos 1988), using a nick-translated MHC gene probe.

\section{RNA isolation, probe preparation, and hybridization}

RNA was isolated from second and third-instar larvae or from late-stage pupae (following eye pigment deposition) by homogenization of 50 organisms in $1 \mathrm{ml}$ of freshly prepared $6 \mathrm{M}$ urea and $3 \mathrm{M} \mathrm{LiCl}$ and incubation overnight on ice. RNA was collected by centrifugation and then resuspended in $300 \mu \mathrm{l}$ of 10 $\mathrm{mM}$ Tris- $\mathrm{HCl}$ (pH 7.5), $10 \mathrm{~mm}$ EDTA, and 1\% SDS. The sample was phenol/chloroform-extracted twice and extracted once with chloroform. RNA was precipitated with ethanol, pelleted by centrifugation, and resuspended in $25 \mu \mathrm{l}$ of sterile distilled water. Ten micrograms of each RNA sample was electrophoresed and blotted as described previously (Zyskind and Bernstein 1989). Prior to blotting, gels were stained with ethidium bromide and observed under ultraviolet light to ensure that equivalent amounts of RNA had been loaded.

Northern blot hybridization probes were ${ }^{32} \mathrm{P}$-labeled antisense RNAs, prepared as described by Melton et al. (1984). To construct the exon 15a- and 15b-containing vectors for exonspecific probe preparation, the BgIII-HindIII fragment encompassing both exons (see Fig. 1A) was cloned into the BamHI and HindIII sites of the pKS vector (Stratagene). By digesting with $P$ vuII to linearize the plasmid and employing T7 RNA polymerase for transcription, an exon 15b-specific probe could be produced. An exon 15a clone was made by inserting the BglII$A v a I$ fragment (see Fig. 1A) into the BamHI site and SmaI site of pKS (the AvaI site was first treated with S1 nuclease to create blunt ends). The exon 15a-specific probe was made by use of $\mathrm{T} 7$
RNA polymerase, following linearization in the polylinker with EcoRI. Northern blot hybridization and washing were essentially as described by DeLeon et al. (1983). Probes labeled with ${ }^{35} \mathrm{~S}$ were prepared for in situ hybridization, which was performed as described previously (O'Donnell et al. 1989).

\section{Light and electron microscopy}

Fixation, embedding, sectioning, and staining of tissues were done as described previously (O'Donnell and Bernstein 1988; O’Donnell et al. 1989).

\section{Acknowledgments}

We thank Roger Sabbadini, Dianne Hodges, and Phillip Singer for their critical comments concerning the manuscript, $\mathrm{Ni}$ cholas Brown for providing the late embryo cDNA library, Eric Fyrberg, Charles Emerson, and Anne Glenn for providing pupal MHC cDNA clones, and Elizabeth George, Margaret Ober, and Charles Emerson for sharing their manuscript with us prior to publication. Our research was supported by grants from the National Institutes of Health (GM-32443) and the Muscular Dystrophy Association. S.I.B. is an Established Investigator of the American Heart Association.

\section{Note added in proof}

Sequence data described in this paper have been submitted to the EMBL/GenBank Data Libraries under accession number $\mathrm{X} 53155$.

\section{References}

Baserga, S.J. and E.J. Benz Jr. 1988. Nonsense mutations in the human $\beta$-globin gene affect mRNA metabolism. Proc. Natl. Acad. Sci. 85: 2056-2060.

Bernstein, S.I., K. Mogami, J.J. Donady, and C.P. Emerson Jr. 1983. Drosophila muscle myosin heavy chain encoded by a single gene in a cluster of muscle mutations. Nature 302: 393-397.

Bernstein, S.I., C.J. Hansen, K.D. Becker, D.R. Wassenberg II, E.S. Roche, J.J. Donady, and C.P. Emerson Jr. 1986. Altemative RNA splicing generates transcripts encoding a thoraxspecific isoform of Drosophila melanogaster myosin heavy chain. Mol. Cell. Biol. 6: 2511-2519.

Brown, N.H. and F.C. Kafatos. 1988. Functional cDNA libraries from Drosophila embryos. J. Mol. Biol. 203: 425-437.

Chun, M. and S. Falkenthal. 1988. Ifm(2)2 is a myosin heavy chain allele that disrupts myofibrillar assembly in the indirect flight muscle of Drosophila melanogaster. J. Cell Biol. 107: 2613-2621.

Crossley, A.C. 1978. The morphology and development of the Drosophila muscular system. In The genetics and biology of Drosophila (ed. M. Ashburner and T.R.F. Wright), vol. 2b, pp. 499-560. Academic Press, London.

DeLeon, D.V., K.H. Cox, L.M. Angerer, and R.C. Angerer. 1983. Most early-variant histone mRNA is contained in the pronucleus of sea urchin eggs. Dev. Biol. 100: 197-206.

Dibb, N.J., D.M. Brown, J.Karn, D.G. Moerman, S.L. Bolten, and R.H. Waterston. 1985. Sequence analysis of mutations that affect the synthesis, assembly and enzymatic activity of the unc-54 myosin heavy chain of Caenorhabditis elegans. I. Mol. Biol. 183: 543-551.

Emerson, C.P., Jr. and S.I. Bernstein. 1987. Molecular genetics of myosin. Annu. Rev. Biochem. 56: 695-726.

George, E.L., M.B. Ober, and C.P. Emerson Jr. 1989. Functional 
domains of the Drosophila muscle myosin heavy chain gene are encoded by alternatively spliced exons. Mol. Cell. Biol. 9: $2957-2974$.

Harrington, W.F. and M.E. Rodgers. 1984. Myosin. Annu. Rev. Biochem. 53: 35-73.

Hattori, M. and Y. Sakaki. 1986. Dideoxy sequencing method using denatured plasmid templates. Anal. Biochem. 152: 232-238.

Hayashi, T., P.M. Wozniak, M.L. Cayer, and D.S. Smith. 1983. Actin-myosin interaction: the role of myosin in determining the actin pattern in self-assembled "hybrid" contractile units. Tissue Cell 15: 955-963.

Hess, N., W.A. Kronert, and S.I. Bernstein. 1989. Transcriptional and post-transcriptional regulation of Drosophila myosin heavy chain gene expression. In Cellular and molecular biology of muscle development. (ed. L.H. Kedes and F.E. Stockdale), pp. 621-631. A.R. Liss, New York.

Hynes, T.R., S.M. Block, B.T. White, and J.A. Spudich. 1987. Movement of myosin fragments in vitro: domains involved in force production. Cell 48: 953-963.

Karn, J., S. Brenner, and L. Barnett. 1983. Protein structural domains in the Caenorhabditis elegans unc-54 myosin heavy chain gene are not separated by introns. Proc. Natl. Acad. Sci. 80: 4253-4257.

Kazzaz, J.A. and C.E. Rozek. 1989. Tissue-specific expression of the alternately processed Drosophila myosin heavy-chain messenger RNAs. Dev. Biol. 133: 550-561.

Kiehart, D.P., D.A. Kaiser, and T.D. Pollard. 1984. Direct localization of monoclonal antibody-binding sites on Acanthamoeba myosin-II and inhibition of filament formation by antibodies that bind to specific sites on the myosin-II tail. $/$. Cell. Biol. 99: 1015-1023.

Kishino, A. and T. Yanagida. 1988. Force measurements by micromanipulation of a single actin filament by glass needles. Nature 334: 74-76.

Kuczmarski, E.R. and J.A. Spudich. 1980. Regulation of myosin self-assembly: Phosphorylation of Dictyostelium heavy chain subunits inhibits formation of thick filaments. Proc. Nat1. Acad. Sci. 77: 7292-7296.

McLachlan, A.D. and J. Karn. 1982. Periodic charge distributions in the myosin rod amino acid sequence match crossbridge spacings in muscle. Nature 299: 226-231.

Melton, D.A., P.A. Krieg, M.R. Rebagliati, T. Maniatis, K. Zinn, and M.R. Green. 1984. Efficient in vitro synthesis of biologically active RNA and RNA hybridization probes from plasmids containing a bacteriophage SP6 promoter. Nucleic Acids Res. 12: 7035-7056.

Mogami, K. and Y. Hotta. 1981. Isolation of Drosophila flightless mutants which affect myofibrillar proteins of indirect flight muscle. Mol. Gen. Genet. 183: 409-417.

Mogami, K., P.T. O'Donnell, S.I. Bernstein, T.R.F. Wright, and C.P. Emerson, Jr. 1986. Mutations of the Drosophila myosin heavy-chain gene: effects on transcription, myosin accumulation, and muscle function. Proc. Natl. Acad. Sci. 83: $1393-1397$.

Mount, S.M. 1982. A catalogue of splice junction sequences. Nucleic Acids Res. 10: 459-472.

O'Donnell, P.T. and S.I. Bernstein. 1988. Molecular and ultrastructural defects in a Drosophila myosin heavy chain mutant: Differential effects on muscle function produced by similar thick filament abnormalities. I. Cell. Biol. 107: 2601-2612.

O'Donnell, P.T., V.L. Collier, K. Mogami, and S.I. Bernstein. 1989. Ultrastructural and molecular analyses of homozygous-viable Drosophila melanogaster muscle mutants indicate there is a complex pattern of myosin heavy-chain isoform distribution. Genes Dev. 3: 1233-1246.
Rozek, C.E. and N. Davidson. 1986. Differential processing of RNA transcribed from the single-copy Drosophila myosin heavy chain gene produces four mRNAs that encode two polypeptides. Proc. Natl. Acad. Sci. 83: 2128-2132.

Smith, C.W.J., E.B. Porro, J.G. Patton, and B. Nadal-Ginard. 1989. Scanning from an independently specified branch point defines the 3 ' splice site of mammalian introns. $\mathrm{Na}$ ture 342: 243-247.

Strehler, E.E., M.-A. Strehler-Page, J.-C. Perriard, M. Periasamy and B. Nadal-Ginard. 1986. Complete nucleotide and encoded amino acid sequence of a mammalian myosin heavy chain gene. I. Mol. Biol. 190: 291-317.

Toyoshima, Y.Y., S.J. Kron, E.M. McNally, K.R. Niebling, C. Toyoshima, and J.A. Spudich. 1987. Myosin subfragment-1 is sufficient to move actin filaments in vitro. Nature 328: $536-539$.

Ueno, H. and W.F. Harrington. 1986. Local melting in the subfragment- 2 region of myosin in activated muscle and its correlation with contractile force. J. Mol. Biol. 190: 69-82.

Wassenberg II, D.R., W.A. Kronert, P.T. O'Donnell, and S.I. Bernstein. 1987. Analysis of the $5^{\prime}$ end of the Drosophila muscle myosin heavy chain gene: alternatively spliced transcripts initiate at a single site and intron locations are conserved compared to myosin genes of other organisms. J. Biol. Chem. 262: 10741-10747.

Zyskind, J.W. and S.I. Bernstein. 1989. Recombinant DNA laboratory manual. Academic Press, San Diego. 


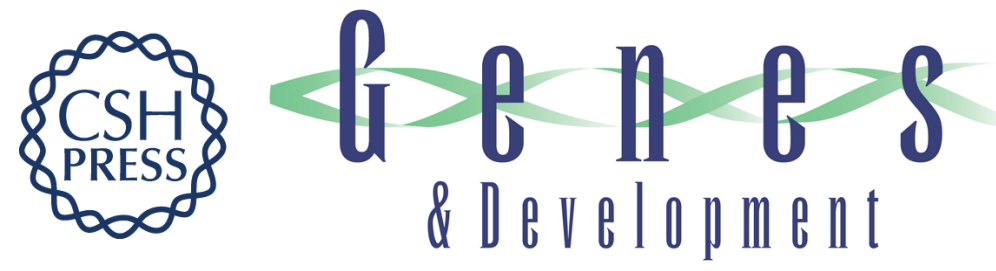

\section{Alternative myosin hinge regions are utilized in a tissue-specific fashion that correlates with muscle contraction speed.}

V L Collier, W A Kronert, P T O'Donnell, et al.

Genes Dev. 1990, 4:

Access the most recent version at doi:10.1101/gad.4.6.885

References This article cites 31 articles, 12 of which can be accessed free at:

http://genesdev.cshlp.org/content/4/6/885.full.html\#ref-list-1

License

Email Alerting

Service

Receive free email alerts when new articles cite this article - sign up in the box at the top right corner of the article or click here.

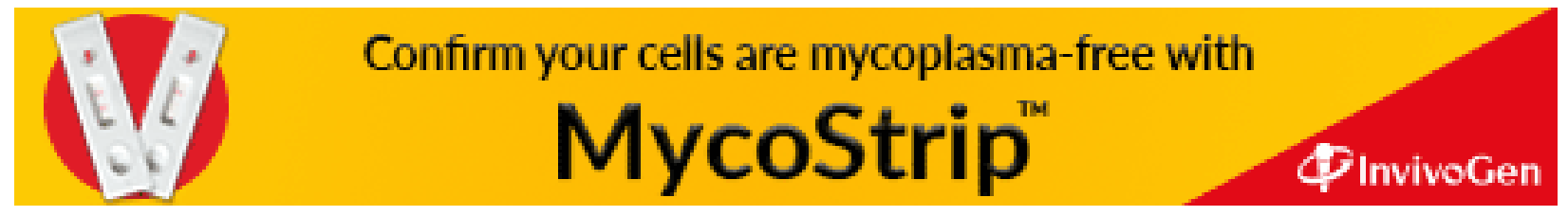

